\title{
artigo
}

Kawakimi, R.M.S.A.; Tomazoni, A.C.; Vanin, C.N.; Souza, F.G.; Pasqualotto, I.; Gattas, M.B.;

Experiências e desafios da formação médica durante a pandemia da COVID-19

\section{Experiências e desafios da formação médica durante a pandemia da COVID-19}

\author{
Experiences and challenges of medical training during the COVID-19 pandemic \\ Experiencias y retos de la formación médica durante la pandemia del COVID-19
}

\begin{abstract}
RESUMO
Objetivo: relatar a experiência e os desafios dos estudantes de medicina do primeiro semestre da formação médica durante a pandemia da COVID-19. Método: Relato de experiência e desafios da graduação médica durante a pandemia do novo coronavírus em uma instituição de ensino superior privada, no período de março a julho de 2020. Resultados: 0 Centro Universitário (UNIVAG) estabeleceu medidas para o enfrentamento da COVID-19, adotando a Educação Domiciliar Emergencial. Essa experiência demonstrou grande capacidade adaptativa dos envolvidos nos processos de aprendizagem dos conteúdos teóricos, bem como atitudes mais criativas dos alunos e a inclusão de outras metodologias ativas no plano de ensino do Programa de Interação Comunitária a fim de aproximar o aluno da realidade. Conclusão: Diante deste cenário, a adaptação para a utilização de tecnologias disponíveis foi um desafio, porém essencial para agregar conhecimento aos graduandos de medicina e continuar 0 ano letivo.
\end{abstract}

DESCRITORES: Infecções por coronavírus; Educação; Medicina.

\section{ABSTRACT}

Objective: to report the experience and challenges of medical students in the first semester of medical training during the COVID-19 pandemic. Method: Report of experience and challenges of medical graduation during the pandemic of the new coronavirus in a private higher education institution, from March to July 2020. Results: The University Center (UNIVAG) established measures to face COVID-19, adopting Emergency Home Education. This experience demonstrated great adaptive capacity of those involved in the learning processes of theoretical content, as well as more creative attitudes of students and the inclusion of other active methodologies in the teaching plan of the Community Interaction Program in order to bring the student closer to reality. Conclusion: Given this scenario, adapting to the use of available technologies was a challenge, but essential to add knowledge to medical students and continue the school year.

DESCRIPTORS: Coronavirus infections; Education; Medicine.

\section{RESUMEN}

Objetivo: reportar la experiencia y desafíos de los estudiantes de medicina en el primer semestre de formación médica durante la pandemia de COVID-19. Método: Informe de experiencia y desafíos de la graduación médica durante la pandemia del nuevo coronavirus en una institución privada de educación superior, de marzo a julio de 2020. Resultados: El Centro Universitario (UNIVAG) estableció medidas para enfrentar el COVID-19, adoptando la educación de emergencia en el hogar. Esta experiencia demostró una gran capacidad adaptativa de los involucrados en los procesos de aprendizaje de contenidos teóricos, así como actitudes más creativas de los estudiantes y la inclusión de otras metodologías activas en el plan docente del Programa de Interacción Comunitaria con el fin de acercar al estudiante a la realidad. Conclusión: Ante este escenario, adaptarse al uso de las tecnologías disponibles fue un desafío, pero fundamental para sumar conocimientos a los estudiantes de medicina y continuar el curso escolar.

DESCRIPTORES: Infecciones por coronavirus; Educación; Medicamento.

RECEBIDO EM: 09/10/2020 APROVADO EM: 27/11/2020

\section{Roselma Marcele da Silva Alexandre Kawakami}

Mestre em Enfermagem. Especialista em Vigilância em Saúde.Especialista em Auditoria dos Serviços de Saúde. Professora de Ensino Superior do Centro Universitário UNIVAG no curso de medicina e enfermagem.

ORCID: 0000-0001-5581-8115 
Ana Carolina Tomazoni

Acadêmica de Medicina do Centro Universitário - UNIVAG.

ORCID: 0000-0003-1388-4530

\section{Camila Natália Vanin}

Acadêmica de Medicina do Centro Universitário - UNIVAG.

ORCID: 0000-0002-3193-0556

Fernanda Gonçalves Souza

Acadêmica de Medicina do Centro Universitário - UNIVAG.

ORCID: 0000-0001-5669-8549

\section{Isabella Pasqualotto}

Acadêmica de Medicina do Centro Universitário - UNIVAG. ORCID: 0000-0002-9850-7285

\section{Marina Bernardes Gattas}

Acadêmica de Medicina do Centro Universitário - UNIVAG.

ORCID: 0000-0002-3886-8169

\section{INTRODUÇÃO}

E ste relato de experiência foi produzido em um contexto de pandemia da COVID-19, pois, no final de dezembro de 2019, em Wuhan, China, foram relatados pacientes com pneumonia viral de causa desconhecida. Após os primeiros casos, o patógeno foi identificado como um novo tipo de Coronavírus, posteriormente chamado de Síndrome Respiratória Aguda Grave Coronavírus 2 (SARS-CoV-2). A patologia associada ao coronavírus 2019 foi descrita como COVID-19, uma pneumonia atípica do tipo SARS $^{1}$. No dia 11 de março de 2020, a Organização Mundial da Saúde (OMS) declarou a pandemia da COVID-19. Até a presente data (31 de julho de 2020), foram relatados 17.296 .303 casos da doença, com registro de 673.290 óbitos ${ }^{2}$.

No Brasil, os primeiros casos confirmados estavam relacionados a pessoas com histórico recente de viagem para áreas epidêmicas e/ou que tiveram contato com casos confirmados ou suspeitos. O Ministério da Saúde, no dia 13 de março de 2020 , constatou a transmissão comunitária, adotando, assim, medidas de distanciamento social com intuito de atenuar a transmissão do SARS-CoV-2 no país. Até o momento, o Brasil apresenta 2.610.102 casos confirmados e 91.263 mortes $^{2}$. A realidade do estado do Mato Grosso é condizente com o atual cenário da pandemia, uma vez que apre- senta uma elevada taxa de transmissibilidade comunitária do vírus, com registro de 48.854 casos confirmados e 1.752 óbitos $^{3}$.

Delimitando para o contexto de interesse deste relato, tem-se a cidade de Várzea Grande, onde se encontra localizado o UNIVAG. A instituição de ensino citada é uma universidade privada, com um projeto pedagógico para a área de ciência médica ancorado no método PBL, sigla de Problem Based Learning, com significado de Aprendizagem Baseada em Problemas, metodologia ativa que incentiva a participação em grupo e a pró-atividade dos integrantes.

Esta cidade está em segundo lugar no rank dos municípios com maior número de casos no estado mato-grossense, com taxa de letalidade de $7,36 \%{ }^{3}$. Isso evidencia a importância das medidas de distanciamento social adotadas pela gestão municipal, que culminaram com a suspensão das aulas presenciais. Inseridos nesse cenário, docentes e discentes enfrentam uma nova realidade no ensino e na formação médica. Para a manutenção e progressão do curso, foram adotados protocolos da Educação Domiciliar Emergencial.

O Programa de Interação Comunitária (PIC) consiste em uma das cinco matérias proposta para o primeiro semestre do curso de medicina do UNIVAG. É mediante o PIC que os acadêmicos são inseridos no cotidiano das Unidades de Saúde da Família e da Comunidade colocando em prática os seus conhecimentos teóricos adquiridos.
Contudo, em virtude da situação pandêmica, novas estratégias tiveram que ser planejadas a fim de alcançar a excelência na disciplina. Sendo assim, o objetivo deste estudo é relatar a experiência e desafios dos estudantes de medicina do primeiro semestre da formação médica durante a pandemia da COVID-19 no contexto do PIC.

\section{MÉTODO}

Trata-se de um relato de experiência de cinco acadêmicas de medicina do primeiro semestre e uma preceptora enfermeira sobre a experiência de cursar a Disciplina do Programa de Interação Comunitária do curso de medicina em uma instituição privada no período de pandemia da COVID-19.

A Instituição de Ensino Superior (IES) privada é localizada em Várzea Grande, cujo curso de medicina utiliza o método de aprendizagem baseada em problema (PBL).

Considerando o surgimento do novo coronavírus, as atividades acadêmicas foram desenvolvidas utilizando especialmente a ferramenta google meet, cujo os encontros das aulas eram online na modalidade ao vivo, mantendo o método da instituição previsto no projeto político pedagógico do curso.

Para coleta das informações foi utilizado o plano de ensino do primeiro semestre e as observações das experiências vivenciadas pelas acadêmicas durante a pandemia no período de março a julho de 2020. 


\section{artigo}

Kawakimi, R.M.S.A.; Tomazoni, A.C.; Vanin, C.N.; Souza, F.G.; Pasqualotto, I.; Gattas, M.B.

Experiências e desafios da formação médica durante a pandemia da COVID-19

Sendo assim, foram analisados os dados seguindo o seguinte percurso:

a) Análise do conteúdo aprendido no plano de ensino.

b) Análise da situação da pandemia do novo coronavírus e as mudanças do modelo presencial para educação domiciliar emergencial.

c) Experiências da formação médica no período de pandemia da COVID-19.

Devido à natureza metodológica dispensa a aprovação do Comitê de Ética em Pesquisa (CEP) de acordo com a resolução $n^{\circ}$ 510/2016 do Conselho Nacional de Saúde (CNS) que dispõe sobre os estudos que não serão submetidos ao CEP.

\section{RESULTADO}

O PIC possui como uma de suas principais finalidades a formação de egressos conscientes da importância da Medicina de Família e Comunidade e da Atenção Básica, além de proporcionar maior compreensão sobre o funcionamento destas áreas no âmbito das Redes de Atenção à Saúde (RAS), que compreendem o Sistema Único de Saúde (SUS).

Para tanto, possui componentes teóricos e práticos que se complementam auxiliando o processo de aprendizagem, já que a prática possibilita a aplicação da teoria, contribuindo para sua plena compreensão e, também, para a inserção do aluno no ambiente de trabalho do profissional de saúde na comunidade, por meio da realização de atividades nas Unidades de Saúde da Família (USF) e suas respectivas áreas de abrangência. Desse modo, a organização das atividades do PIC se dá por meio da divisão da classe em grupos de geralmente cinco alunos, sendo que cada um é supervisionado e orientado por um preceptor (a) e alocado em uma USF.

Nesse contexto, com o início do ano letivo, que ocorreu ao final de fevereiro, as primeiras aulas dessa disciplina seguiram normalmente as atividades conforme o calendário estipulado.

Entretanto, no dia 17 de março a universidade optou pela suspensão das aulas presenciais, visando à segurança de todos em meio à pandemia da COVID-19. Com isso, o corpo docente responsável pelo PIC desenvolveu novas estratégias de ensino frente ao novo contexto, buscando maneiras de transmitir o conhecimento necessário, além de promover o engajamento dos discentes à distância, tendo por objetivo evitar o atraso do calendário acadêmico, dando continuidade às aulas.

Nesse sentido, foi implementada, no dia 17 de abril, a Educação Domiciliar Emergencial, a qual deu segmento aos conteúdos teóricos, por meio da utilização das plataformas digitais de videoconferência e Google Meet, mantendo o aluno em contato relativamente direto e instantâneo com o professor para que pudessem, especialmente, esclarecer dúvidas durante a ministração dos conteúdos. Dessa forma, ocorreu o remanejamento da execução das atividades práticas para o momento de retorno às aulas presenciais.

Para a realização das atividades teóricas, juntamente às plataformas de videoconferência, foi utilizada a plataforma do Ambiente Virtual de Aprendizagem (AVA/UNIVAG), esse dispositivo já constava como material de apoio para as disciplinas nas quais os alunos estavam inscritos, onde eram postadas informações referentes ao calendário acadêmico de cada semestre. Entretanto, com a paralização das atividades presenciais o AVA passou a ser de fundamental importância para a disponibilização dos recursos didáticos referentes, principalmente, à disponibilização de materiais de estudo relativos a cada disciplina, fato evidenciado por alunos e professores.

Tabela 1 - Conteúdos teóricos do primeiro semestre do curso de medicina na disciplina do Programa de Interação Comunitária. Várzea Grande- MT, 2020.

$$
\text { Tema }
$$

Sistema Único de Saúde

Rede de Atenção a Saúde

Política Nacional de Atenção Básica

Atenção Domiciliar e ferramentas utilizadas

Processos de educação para atuação nos serviços de saúde 1

Assistência a saúde da pessoa indígena

Resíduos sólidos dos serviços de saúde

Indicadores de saúde

Total 1 
cimento por meio de exemplos, esclarecimento de possíveis dúvidas, mas principalmente por meio da elaboração e discussão de estudos de caso clínicos. Esse último consistiu em uma das estratégias adotadas pela disciplina para tornar o conteúdo menos abstrato e aprimorar a compreensão a respeito do tema abordado.

Assim, ao final de cada discussão teórica, a preceptora apresentou um caso clínico de acordo com o tema daquele dia, em seguida realizou questionamentos pertinentes a respeito das condutas a serem tomadas de acordo com o que foi observado. Esses questionamentos seguiram de maneiras diversas, tanto na forma de questóes abertas quanto objetivas, o que acabou por simular o processo avaliativo que ocorreu ao final do semestre.

Além desses métodos, certas vezes, também foi disponibilizado via AVA, listas de exercícios para a fixação do conteúdo aprendido, os conteúdos e atividades previstas para o semestre.

A forma da avaliação adotada pela universidade durante a pandemia consistiu nas notas formativa e somativa, ambas com pesos equivalentes e que somadas compõem a nota semestral. A primeira consiste na avaliação individual do aluno bem como de sua interação com o grupo durante o desenvolvimento das discussões teóricas, já a segunda ocorre por meio da realização de uma prova, a qual pode seguir o modelo tradicional físico ou oral.

Considerando os parâmetros anteriores à pandemia, a avaliação formativa permaneceu inalterada, já a somativa necessitou alterações, pois era realizada presencialmente, ao modelo tradicional, com questões objetivas e discursivas. Sendo assim, a prova foi realizada no modelo oral, utilizando a plataforma de videoconferência, em que cada aluno a realizou individualmente com seu preceptor, com câmera e áudio abertos e tendo as questões projetadas por esse mesmo instrumento.

O conteúdo da avaliação era referente ao que foi abordado durante as discussões teóricas e conferências, ao qual caberia estudo prévio para que no dia da avaliação respondesse às perguntas abertas e objetivas que eram projetadas, permitindo a reflexão do aluno sobre o conteúdo. Cabe ressaltar que o processo avaliativo ocorreu sem maiores dificuldades, visto que seguiu o modelo das simulações realizadas após as discussões.
Dessa forma, o primeiro semestre do curso foi concluído, mas não sem empecilhos, como problemas de conexão com a internet, o que, por vezes, atrapalhava o fluxo de comunicação, entretanto, com a paciência e compreensão de todos, tal questão foi superada com tranquilidade, não prejudicando significativamente $\mathrm{o}$ aprendizado.

Outrossim, a ausência da prática também dificultou o processo de aprendizagem, visto que, aprender sobre uma profissão sem o efetivo contato com a mesma torna-se, de fato, uma experiência mais desafiadora. Somado a isso, o incentivo à produção científica, que geralmente ocorre desde o início da formação acadêmica, devido às construções de relatos de experiências relacionados a prática na USF ficou comprometido, visto que a grande maioria das iniciativas ocorriam presencialmente, por meio de reuniões, debates e esclarecimentos sobre temática e confecção.

Em contrapartida, uma das estratégias adotadas pelo grupo que auxiliou a dinâmica das discussões, foi à exploração dos recursos digitais disponíveis para a confecção e apresentação na plataforma de videoconferência de resumos, slides utilizando power point ou prezi, mapas mentais e imagens sobre as temáticas propostas, os quais eram projetados durante as discussões teóricas, servindo de suporte para o aprendizado; essas atitudes foram mais presentes durante a pandemia, o que demonstrou maior criatividade dos alunos.

Nesses casos, os materiais eram elaborados conjuntamente pelas discentes, com base em discussões prévias realizadas por meio de aplicativos de mensagem instantânea, onde planejavam o seguimento da discussão teórica para que todos os pontos importantes recebessem o devido destaque, sendo que também debatiam entre si as suas dúvidas, em um processo de ajuda mútua. Além disso, pelas mesmas ferramentas de comunicação instantânea, criou-se um grupo de whatsapp juntamente à preceptora, o qual também serviu como meio de auxílio para as comunicações que precisavam ser feitas (como recados e aviso) e possíveis outras dúvidas que surgissem, o que acabou por aproximar o contato entre todos os integrantes.

Diante do exposto, constata-se que mesmo sendo um período de grande dificulda- de, pois a permanência em casa, por vezes, requer esforço adicional para concentração e muita disciplina, a dedicação dos alunos nesse período foi crucial para o bom funcionamento deste modelo de educação emergencial, considerando a grande participação do corpo discente na metodologia ativa que compõe a base do PBL, fazendo com que o conteúdo acadêmico proposto fosse realizado e compreendido adequadamente, destacando que a comunicação e criatividade foram atitudes que foram destacadas no contexto de pandemia, sendo explorada e apresentando-se como diferencial no comportamento dos alunos.

Por outro lado, a dedicação da preceptora do grupo foi imprescindível para a superação desse desafio, possibilitando o desenvolvimento e aprendizado de seus alunos durante o seguimento deste semestre excepcional, visto que, os professores enfrentaram o desafio de aprender uma nova metodologia de ensino, o uso das plataformas e, ao mesmo tempo, tranquilizar e estimular seus alunos constantemente, se preocupando com as emoções, aprendizagem e satisfação com o curso, docente e instituição.

\section{DISCUSSÃO}

A formação médica de inúmeros indivíduos foi afetada por conta da pandemia causada pelo SARS-CoV-2, contudo, os principais envolvidos nessa problemática foram os universitários do curso de medicina. Esses acadêmicos foram submetidos a ações emergenciais advindas das universidades que implementaram a educação domiciliar emergencial.

A modalidade de ensino remoto emergencial (ERE) não se resume apenas em aulas online, mas, sim, em uma metodologia mais ativa do aluno com o seu próprio aprendizado, através de estudos prévios, discussões teóricas entre os discentes e maior contato com os educadores. Em contrapartida, o Ensino a Distância (EAD) como estrutura de aprendizado possui uma proposta diferente, visto que há a oferta apenas de aulas em ambientes virtuais sem a participação ativa dos estudantes4. Alguns centros acadêmicos, como o UNIVAG, apresentaram uma experiência positiva sobre essa metodologia aplicada para 


\section{artigo}

conteúdos teóricos, com ótimos desempenhos avaliativos e contiguidade na adesão de conhecimento pelos discentes.

Apesar dos benefícios do ERE, de acordo com o estudo desenvolvido pela Universidade Católica de Portugal que fez um comparativo entre as Escalas de Ansiedade, Depressão e Stress (EADS-21) dos anos de 2018, 2019 e o período atual de pandemia, verificou-se que houve um aumento significativo de perturbação psicológica entre os acadêmicos no período pandêmico, pois enfrentaram alguns obstáculos com a aplicação do ERE, como o aumento nos níveis de depressão, ansiedade e estresse, o que pode acabar reduzindo o desempenho estudantil ${ }^{5}$.

Trabalhar as emoções dos alunos e auxiliar no processo de amadurecimento emocional é uma medida de extrema importância, para que essa queda no desempenho não seja observada. Segundo estudo, o principal responsável por auxiliar nessa evolução deve ser o educador, e para que isso seja possível, promover reflexões e análises é necessário6 em cenários conflitantes, como o atual.

Sob outro viés, no Centro Universitário UNIVAG, as modalidades práticas, em oposição às teóricas, foram paralisadas no ciclo básico (primeiro e segundo ano). Apesar de ser uma ação responsável e necessária para o atual contexto de pandemia, também são observadas fragilidades por conta dessa interrupção, a falta das práticas dificulta a associação da aplicabilidade dos conhecimentos teóricos apreendidos, a fim de minimizar os danos o UNIVAG desenvolveu estudos de casos simulando uma realidade, com a finalidade de aproximar os estudantes do contexto real do serviço de saúde.

A literatura corrobora, apontando outra desvantagem do ERE, sendo destacado o aumento na espera dos graduandos para serem inseridos no mercado de trabalho, o que pode acarretar problemas, como desistência do curso e perda de renda. Outro prejuízo é quanto à reposição desses procedimentos práticos perdidos, que tem sua data de implementação indeterminada, gerando inúmeras incertezas entre os estudantes quanto ao padrão de qualidade que essas aulas possuirão ${ }^{7}$.

Além disso, vale ressaltar, que as doenças com alta taxa de transmissibilidade, como a COVID-19, exigem que acadêmicos e profissionais da saúde reforcem as medidas de proteção através do uso de EPI, para que a contaminação em massa seja evitada. Contudo, em países como Turquia e Brasil, esse aumento no cuidado não se apresenta plenamente possível, devido à redução no acesso a essas ferramentas de proteção. No caso da Turquia, estudo relata que apesar da oferta de EPI, como máscaras, serem uma obrigatoriedade do governo, cidadãos civis e também profissionais da saúde estão tendo dificuldades em adquirir esses equipamentos gratuitamente, isso se deve ao fato de as ações governamentais não terem sido suficientes para atender a demanda da população turca, o que corrobora para a queda na acessibilidade desses equipamentos no país ${ }^{8}$.

Já no Brasil, uma das causas dessa diminuição no acesso desses EPI, é o aumento exorbitante na demanda por esses equipamentos de proteção cirúrgicos utilizados pelos profissionais e acadêmicos, como as máscaras Peça Facial Filtrante do tipo 2 (PFF2) e respirador de partículas N95. Essa problemática foi gerada por conta de uma parte da sociedade civil ter estocado esse tipo de EPI em suas casas, e, consequentemente uma diferença numérica entre a produção e o consumo desses equipamentos acaba sendo gerada?.

Os profissionais e acadêmicos da área da saúde são os mais afetados por essa questão, já que ter acesso a esses EPI é crucial para um atendimento e estudo com segurança. Além disso, o subfinanciamento do SUS no Brasil10 também acentua essa problemática de redução na infraestrutura médica, o que também pode gerar um aumento no número de infectados pela doença.

Essas perspectivas deixam explícito que o retorno de atividades práticas pelo ciclo básico seria mais desafiador no momento de ritmo acelerado de crescimento de casos da COVID-19, mesmo com a disponibilização de EPI pela instituição. Sendo assim, a instituição sabidamente manteve-se em educação domiciliar emergencial preparando seus alunos por meio de conteúdo teórico, assim como aulas de biossegurança para preparar os acadêmicos para um retorno seguro.

Ademais, outro desafio, na formação médica de acadêmicos do ciclo básico do
UNIVAG, foi à redução de algumas atividades ofertadas pela comunidade científica. A participação ativa, desde o início da carreira de seus estudantes nessas modalidades, sempre foi estimulada por meio de disciplinas essenciais como o PIC.

A literatura mostra que uma das principais atividades que foram comprometidas por esse período pandêmico foram os experimentos científicos. Isso se deve ao fato de que a comunidade científica foi obrigada a paralisar a realização desses projetos e consequentemente houve um atraso no desenvolvimento de experimentos e perda de cronogramas. Alguns pesquisadores contornaram essa situação conflituosa dando continuidade nos projetos, porém em casa através de modernas tecnologias. Em alguns casos essa modalidade de pesquisa se tornou um mecanismo positivo, não somente na formação médica, mas também de outros cursos, como expõe o pós-doutorando do segundo ano na Universidade Agrícola de Huazhong, Wuhan China que teve ótimas experiências com o desenvolvimento de seus projetos através de plataformas virtuais. Contudo, essa questão também pode se tornar um empecilho para alguns indivíduos, como o caso de Bai Zheng, mestre estudante da Universidade de Zhejiang, Hangzhou China que se sentiu uma regressão de seus projetos desenvolvidos em casa $^{11}$.

O cancelamento de eventos científicos, como simpósios, conferências e oficinas, também agravaram o contexto de formação médica. Isso se deve ao fato de que, grande parte do conhecimento, adquirido durante a realização de projetos e experimentos, é disseminado aos demais indivíduos através da realização desses eventos. Com esse cancelamento, inúmeras ideais e perspectivas deixaram de ser propagadas, além disso, prejuízos econômicos também foram gerados, o que afeta os organizadores e participantes desses eventos. A realização de conferências virtuais é um desafio por causa das diferenças de fuso horário, no caso de eventos internacionais, e também a redução da acessibilidade, já que o acesso à internet é uma exigência para que o indivíduo usufrua do conhecimento. Outra perspectiva que também afeta comunidade científica é a redução de investimentos e financiamentos 
de projetos devido às incertezas econômicas geradas pela pandemia. Consequentemente há uma diminuição do desenvolvimento de projetos já iniciados e também que possivelmente seriam admitidos ${ }^{12}$.

Portanto, entende-se que as experiências que são desenvolvidas na atenção primária subsidiam a formação médica, especialmente o que tange o desenvolvimento de competências relacionadas à educação em saúde ${ }^{13}$.

\section{CONCLUSÃO}

Diante desse cenário apresentado é notório que a cooperação, a adaptação e os traços de novas estratégias por parte dos discentes, docentes e o UNIVAG foram fatores que permitiram a execução favorável do plano de educação domiciliar emergencial e a incorporação do conhecimento, respeitando a saúde não só dos envolvidos no processo de educa- ção, mas também de toda a população local, a favor das medidas protetivas.

É visível ainda que a metodologia ativa proposta, o método PBL, foi essencial para fortalecer a continuidade de aprendizado proposto durante a pandemia. Corrobora para essa afirmação o fato de que as discussões teóricas da disciplina PIC foram agregadoras e favoreceram a acumulação de conhecimento por todos os alunos.

Alguns empecilhos foram encontrados durante o semestre como as dificuldades técnicas com conexão de rede, ausência de aulas práticas e o cancelamento de alguns eventos científicos que auxiliam no contexto da formação médica, foram suspensos por universidades, como o UNIVAG, seguindo os protocolos de distanciamento social do Ministério da Saúde.

Apesar das circunstâncias, a universidade tem planos para fazer a reposição dessas aulas práticas como forma de aprendizado prático, sendo esse um desafio ainda a ser superado, todavia os alunos já estão sendo preparados para um retorno seguro por meio de conferências de biossegurança. Quanto aos eventos, os encontros cabíveis à modalidade virtual estão sendo realizados de forma a agregar conhecimento aos graduandos.

Apesar das prerrogativas, o primeiro semestre do curso de medicina no UNIVAG foi concluído com êxito, destacando que em meio à pandemia os acadêmicos demonstraram mais atitudes criativas comparadas ao período anterior, se adaptando à nova modalidade de ensino proposta. Destaca-se, por fim, a importância da Educação Domiciliar Emergencial para o prosseguimento do ano letivo dos graduandos da rede privada, assegurando a saúde comunitária enquanto perdurar o contexto da pandemia da COVID-19.

\section{REFERÊNCIAS}

1. Huang C, Wang Y, Li X, Ren L, Zhao J, Hu Y, et al. Clinical features of patients infected with 2019 novel coronavirus in Wuhan, China. Lancet. 2020 Feb;395(10223):497-506.

2. European Centre for Disease Prevention and Control. An agency of the European Union. COVID-19 Situation Update Worldwide, as of 31 July 2020. [Internet]. [cited 2020 jul 31] Available from: https://www.ecdc.europa.eu/en/geographical-distribution-2019-ncov-cases

3. Governo de Mato Grosso. Boletim epidemiológico n. 143. Situação epidemiológica. 29 de Julho de 2020. Available from: http://www.mt.gov.br/documents/21013/0/Boletim+Informativo $+n \% C 2 \% B A+143 / a 5 c e 1101-7 e 25-b f c 1-b c 0 a-c 75 f 5 f f 3 d 6 f 8$

4. Todos pela Educação. Nota técnica. Ensino a distância na educação básica frente à pandemia da covid-19. Análise e visão do Todos Pela Educação sobre a adoção de estratégias de ensino remoto frente ao cenário de suspensão provisória das aulas presenciais. Abril, 2020. Available from: https:// www.todospelaeducacao.org.br/_uploads/_posts/425.pdf?1730332266=\&utm_source=conteudo-nota\&utm_medium=hiperlink-download

5. Maia BR, Dias PC. Anxiety, depression and stress in university students: The impact of COVID-19. Estud Psicol [Internet]. 2020 [cited 2020 Aug 1];37. Available from: http://www.scielo.br/scielo. php?script=sci_arttext\&pid=S0103-166X2020000100504\&Ing=en\&nrm=iso\&tlng=pt

6. Blasco PG. Benedetto MAC. Levites MR. Moreto G. Janaudis MA. Vista do Humanismo e Educação Médica em Tempos de COVID-19. Rev Med (São Paulo). 2020 mar.-abr.; 99(2):i-vi. [Internet]. [cited 2020 Aug 5]. Available from: http://www.revistas. usp.br/revistadc/article/view/169001/160261

7. Rajgor DD, Lee $H$, Archuleta S, Bagdasarian N, Quek SC. COVID-19 and medical education. Lancet Infect Dis [Internet]. 2020 [cited 2020 Aug 1];20:777-8. Available from: https:// gisanddata.maps.arcgis.

8. Aydogdu ALF. Pandemia ocasionada pelo novo Coronavírus: sistema de saúde e medidas de enfrentamento na Turquia. J. nurs. health. 2020;10 (n.esp.):e20104008

9. Meneses AS. Gerenciamento Emergencial de Recursos da Atenção Primária a Saúde no Enfrentamento a pandemia da COVID-19. SciELO Preprints, 2020. DOI: https://doi.org/10,1590/ SciELOPreprints.557.

10. Andrade RO. Covid-19 is causing the collapse of Brazil's national health service. The BM] [Internet]. 2020 Jul 30 [cited 2020 Aug 1];370:m3032. Available from: http://www.bmj.com/lookup/doi/10.1136/bmj.m3032.

11. Cheng C. Song S. How Early-Career Researchers Are Navigating the COVID-19 Pandemic. Mol Plant [Internet]. 2020 [cited 2020 Aug 1]; Available from: https://doi.org/10.1016/j. molp.2020.07.018.

12. Subramanya SH, Lama B, Acharya KP. Impact of COVID-19 pandemic on the scientific community, Qatar Medical Journal 2020:21 http://dx.doi.org/10.5339/ qmj.2020.21

13. Santos FF. Miranda CZ. Pertilell KC. Barbosa MS. Caldeira AP. Costa SM. Desempenhos na Área de Competência Educação em Saúde: Autoavaliação de Estudantes de Medicina. Revista Brasileira de Educação Médica. 44 (3): e085; 2020. DOI: https://doi. org/10.1590/1981-5271v44.3-20190291 